\title{
Advanced Optimization Models For Bandwidth Provisioning And Routing In Fixed Microwave Backhaul Networks
}

Djamil Mehadji ( $\sim$ djam.mehadji@gmail.com )

University of Oran 1 https://orcid.org/0000-0002-0679-9867

Mejdi Kaddour

University of Oran 1

\section{Research Article}

Keywords: fixed microwave networks, network capacity dimensionning, minimum cost multicommodity flows, mixed-integer linear programming, Lagrangian relaxation

Posted Date: December 29th, 2021

DOI: https://doi.org/10.21203/rs.3.rs-1100391/v1

License: (c) (1) This work is licensed under a Creative Commons Attribution 4.0 International License.

Read Full License 


\title{
Advanced Optimization Models for Bandwidth Provisioning and Routing in Fixed Microwave Backhaul Networks
}

\author{
Djamil Mehadji ${ }^{*}$ and Mejdi Kaddour ${ }^{1}$ \\ ${ }^{1 *}$ LITIO Laboratory, Department of Computer Science, University of Oran 1, Oran, \\ 31000, Algeria.
}

*Corresponding author(s). E-mail(s): mehadji.djamil@edu.univ-oran1.dz;

Contributing authors: kaddour.mejdi@univ-oran1.dz;

\begin{abstract}
This paper addresses the problem of determining bandwidth allocation and traffic routes in fixed microwave networks such that overall bandwidth cost is minimized while traffic demands are satisfied with a required reliability level. These networks exhibit high variability in link throughput as modulations schemes are adapted dynamically to ensure acceptable bit-error rate at the receivers according to external conditions such as the weather. First, we formulate an optimal optimization approach based on mixed-integer linear programming, which is subsequently reinforced by inserting problem-specific valid inequalities based on global network capacity and feasible bandwidth/modulation combinations. Then, we introduce a Lagrangian-based heuristic that provides near optimal solutions while reducing drastically the computation time. In comparison to previous work, our experimental results show that our approaches are capable to solve large real-world network instances in an effective manner. Furthermore, the results evaluate the impact of reliability and transported traffic demands on bandwidth cost.
\end{abstract}

Keywords: fixed microwave networks, network capacity dimensionning, minimum cost multicommodity flows, mixed-integer linear programming, Lagrangian relaxation

\section{Introduction}

As a consequence of tremendous advances in radio access technologies, the capacity bottleneck of mobile networks is gradually shifting from the radio interface towards the backhaul portion of operator's infrastructures. In this area, microwave networks are increasingly viewed by decisions makers and network architects as a viable alternative to costly fiber-based wired backhaul, in particular in large geographical regions with low density, as they provide cost-effective and easy-todeploy transmission links.
Microwave networks are formed by a set of terrestrial point-to-point radio links using directional antennas in clear line-of-sight and operating in licensed frequency bands [1]. Data rate on each link depends on the used bandwidth and the modulation scheme of the carrier signal. However, the experienced performance of each modulation scheme in terms of bit-error ratio (BER) might be quite variable as it depends heavily on weather conditions. Rain or snow falls have a significant impact on the signal quality by deteriorating the BER at the receiver. Recent advances in wireless technologies enable microwave transceivers to adjust dynamically their modulation scheme, either by decreasing the number of bits per symbol 
in degraded channel conditions, or by increasing it again once these conditions have been improved.

One major challenge in deploying microwave networks is dimensioning as it drives both the achievable throughput and the incurred financial cost. One pre-existing transmission facilities, this task mainly consist in allocating the optimal amount of bandwidth on each link. One has to take in consideration traffic patterns between endsites that may cross multiple hops. Due to the variability of experienced data rates as stated earlier, a relevant optimization problem here is to allocate bandwidth and devise routing scheme so that traffic requests are provisioned with a prescribed level of reliability while reduced the cost associated with bandwidth licensing.

However, as shown in previous work such as [2], this allocation and routing problem constitutes an NP-hard problem. Our aim in this paper is to provide both optimal and heuristic solution approaches able to solve efficiently real-world instances. This is particularly crucial in settings where traffic rerouting to overcome link outages or demand modifications must be carried in short amount of time to minimize service disruption. In particular, we employ following methods to tackle this problem:

- design a mixed-integer linear program (MILP) providing optimal solutions;

- reinforce the original model by adding valid inequalities and eliminate unfeasible settings in order to reduce the search space;

- design a heuristic approach operating in two phases: (i) derive a lower bound on the optimal solution using Lagrangian relaxation; (ii) transform this bound to a feasible solution by a special-purpose algorithm that tweaks provisioned bandwidth, traffic routes, or downscale accepted demands.

This remainder of this paper is structured as follows. In Section 2, we discuss and analyze various contributions related to our work. In Section 3 , we detail the basic formulation of our problem as an MILP. Section 4 is devoted to model reinforcement through global capacity inequality and elimination of unfeasible bandwidth/modulation combinations. In Section 5, we discuss our Lagrangian-based approach to solve the problem in an effective manner. Section 6 reports results and analysis on various numeric experiments targeting the performance of introduced models and the impact of reliability and traffic demands on cost. Some final remarks and comments on future work are given in the final section.

\section{Related Work}

A number of optimization models have been proposed in the literature to tackle capacity planning and dimensioning of microwave wireless networks. These models fall generally within the theoretical category of MCMCF (minimum cost multicommodity flows) problems which have been approached in different manners and applied in multiple application domains. Authors in $[3,4]$ aimed to minimize the overall network cost, made up by installation costs and bandwidth-licensing fees, while ensuring the transportation of a set of commodity flows from sources to destinations node, eventually passing through a set of intermediate nodes.

Some work focused on designing strong models to derive optimal dimensioning. Proposals as in [5-8] reinforced a basic model adding validinequality, in order to reduce the search space in an attempt to tackle large instances. In [7], authors exploited robust optimization techniques to handle the variability of traffic demands. Their approach is enhanced by introduced several specific valid-inequalities. Five classes of these inequalities have been considered in [8] using a cutting plane algorithm.

Another class of papers dropped the optimality requirement by designing very fast polynomial procedures to obtain high-quality solutions. The authors in [9] proposed an iterative linear relaxation-based heuristic which fixes the maximum number of binary variables after each solution of the relaxed problem. In [10], the authors compared the subgradient method and bundle methods to find the best bound.

A number of other papers targeted minimumcost bandwidth provisioning while guaranteeing a certain level of reliability (e.g., original traffic demands are delivered in $99 \%$ of the time). In [11], the authors formulated a chance-constrained mathematical program for fixed broadband wireless networks under unreliable channel conditions. 
The authors in [12] proposed a methodology to compute the exact reliability of a backhaul wireless network and to optimize its design given a limited budget. Finally, Kodjo et al. [13] worked on an optimization model to dimension a backhaul microwave network while considering an dynamic routing scheme. Based on a column-generation framework, the initial problem is divided into two sub-problems: a master problem and a pricing problem. The solution consists in finding a set of transmission scenarios (e.g., current modulation scheme on each link) that have a cumulative probability of occurrence greater or equal to a given reliability threshold.

In comparison, our main objective is the present paper is to explore different solution approaches, either optimal or heuristic, in order to tackle efficiently large network instances which have not been addressed previously.

\section{Optimization Model}

We represent the network topology as a directed graph $G=(V, L)$, where each node $v \in V$ designates a radio node and each link $\ell \in L$ represents a microwave link from the source node $o(\ell)$ to the destination node $d(\ell)$, with $o(\ell), d(\ell) \in V \cdot \omega^{+}(v)$ denotes the set of outgoing links, while $\omega^{-}(v)$ denotes the set of inbound links of $v$. Besides, $B$ and $M$ refer to the sets of possible modulations and bandwidths, respectively. The set of possible amounts of bandwidth for $\ell \in L$ is denoted by $B_{\ell} \subseteq B$, whereas the set of applicable modulations for a given link and bandwidth pair is denoted by $M_{\ell}^{b} \subseteq M$. With a slight abuse of notation, $m \in M$ means also in this paper the number of different symbols of an m-QAM modulation scheme.

Our optimization model est mainly based on the decision binary variables $a_{\ell}^{b m}$ indicating whether the link $\ell$ with bandwidth $b$ and modulation $m$ forms part of the optimal solution setup. Constant Cost $_{b}$ denotes the financial cost of bandwidth $b$, whereas $\theta_{b m}$ denotes the achievable data rate on link of bandwidth $b$ if a acceptable modulation $m$ is used.

Assuming that each transmitter implements some mechanism to adjust its current modulation depending on experienced channel conditions, we designate by $P_{\ell}^{b m}$ the probability that traffic on link $\ell$ with bandwidth $b$ and modulation $m$ is carried with an acceptable bit error rate. Note that
$P_{\ell}^{b m} \geq P_{\ell}^{b m^{\prime}}$ if $m^{\prime}$ is a stronger modulation that $m$ (e.g., $m$ is 16 -QAM and $m^{\prime}$ is 32-QAM). $F$ is the required level of system reliability corresponding to the fraction of initial traffic demands delivered successfully to their intended recipients. Note that we assume in this paper that no dependency exists between link conditions, hence the overall system reliability can be written as the product of link probabilities over $L$.

Traffic demands are modeled by matrix $D=$ $\left(D_{s d}\right)$, where $D_{s d}$ denotes the amount of traffic sent from node $s$ to node $d$. SD designate the set of source-destination pairs. The flow variable $\varphi_{s d}$ indicates the portion of $D_{s d}$ routed through link $\ell \in L$. Using all the previous notation, our optimization model can be stated as follows.

$$
\min \sum_{\ell \in L} \sum_{b \in B_{\ell}} \sum_{m \in M_{\ell}^{b}} \operatorname{Cost}_{b} a_{\ell, b m}
$$

subject to:

$$
\sum_{\ell \in \omega^{-}(v)} \varphi_{\ell}^{s d}-\sum_{\ell \in \omega^{+}(v)} \varphi_{\ell}^{s d}=\left\{\begin{array}{cc}
-D_{s d}, & \text { if } v=s \\
D_{s d}, & \text { if } v=d \\
0, & \text { otherwise }
\end{array}\right.
$$

$$
v \in V,(s, d) \in \mathcal{S D}
$$

$$
\sum_{(s, d) i n \mathcal{S D}} \varphi_{\ell}^{s d} \leq \sum_{b \in B_{\ell}} \sum_{m \in M_{l}^{b}} \theta_{b m} a_{\ell, b m} \quad \ell \in L
$$$$
\sum_{m \in M_{l}^{b}} a_{\ell, b m} \leq 1
$$

$\sum_{\ell \in L} \sum_{b \in B_{\ell}} \sum_{m \in M_{\ell}^{b}} \log \left(P_{\ell}^{b m}\right) a_{\ell, b m} \geq \log (F)$

$$
\begin{array}{lr}
\varphi_{\ell}^{s d} \geq 0 & \ell \in L,(s, d) \in \mathcal{S D} \\
a_{\ell, b m} \in\{0,1\} & \ell \in L, b \in B_{\ell}, m \in M_{\ell}^{b}
\end{array}
$$

where the objective function (1) minimizes the operational cost of the network associated with aggregate licensed bandwidth. Constraints (2) ensure flow conservation from sources to destinations. Constraints (3) guarantee that total traffic transported on each link doesn't exceed its capacity. Constraints (4) restrict each link to a single bandwidth/modulation pair. Constraints (5) ensure that the desired reliability is achieved. 
Note that the logarithmic functions are used to linearize the original constraint written as the product of independent probabilities $P_{\ell}^{b m}$ over the activated transmission links.

\section{Reinforcement of the Original Model}

The aforementioned optimization model is based on a mixed-integer linear program, known to be hard to solve in the general case. In particular, the number of binary grows according to $|E| \times|B| \times$ $|M|$. In this section, we strengthen this model with a set of valid-inequalities in order to reduce the feasible solution space and hence speedup the resolution process. First, we characterize the global capacity of our network.

\subsection{Global capacity}

Let us refer to the global capacity of the network by $\theta_{G}$. From the previous model, it can be written as:

$$
\theta_{G}=\sum_{\ell \in L} \sum_{b \in B_{\ell}} \sum_{m \in M_{l}^{b}} \theta_{b m} a_{\ell, b m}
$$

Also, let $\mu_{G}$ denotes the cumulative total flow carried by the network, written as

$$
\mu_{G}=\sum_{\ell \in L} \sum_{(s, d) \in \mathcal{S D}} \varphi_{\ell}^{s d}
$$

By summing up constraints (3) over links in $L$ we have:

$$
\mu_{G} \leq \theta_{G}
$$

Now we formulate the minimal cumulative total flow $\hat{\mu}_{G}$ that fulfills flow conservation constraints (2) while ignoring actual link capacities.

Lemma $1 \hat{\mu}_{G}=\sum_{(s, d) \in \mathcal{S D}} \rho_{s d} D_{s d}$, where $\rho_{s d}$ denotes the length of minimum-hop path between $s$ and $d$.

Proof The amount of flow generated by demand $D_{s d}$ between node $s$ and node $d$ is $\sum_{\ell \in L} \varphi_{\ell}^{s d}$. According to constraints (2): $\sum_{\ell \in \omega^{-}(v)} \varphi_{\ell}=\sum_{\ell \in \omega^{+}(v)} \varphi_{\ell}^{s d}$ for $v \notin\{s, d\}$, and

$\sum_{\ell \in \omega^{-}(v)} \varphi_{\ell}^{s d}=D_{s d}$ for $v=d$.

Assume that demand $D_{s d}$ is routed over paths with equal lengths $n$, and let $\Gamma_{s d, n}^{i}$ refers to the set of nodes reached after the $i^{t h}$ hop on these paths, then:

$$
\begin{array}{r}
\sum_{v \in \Gamma_{s d, n}^{i}} \sum_{\ell \in \omega^{-}(v)} \varphi_{\ell}^{s d}=\sum_{v \in \Gamma_{s d, n}^{i+1}} \sum_{\ell \in \omega^{-}(v)} \varphi_{\ell}^{s d} \\
i \in 1 \ldots n-1
\end{array}
$$

and

$$
\sum_{v \in \Gamma_{s d, n}^{n}} \sum_{\ell \in \omega^{-}(v)} \varphi_{\ell}^{s d}=D_{s d}
$$

Adding up these equalities, the cumulative flow generated in the network by this demand is $\sum_{\ell \in L} \varphi_{\ell}^{s d}=n D_{s d}$.

Replacing $n$ by $\rho_{s d}$, the lowest possible value for each demand, and summing up, we obtain the result.

Finally, from (10), we add the following valid inequality to strength our original model

$$
\sum_{(s, d) i n \mathcal{S D}} \rho_{s d} D_{s d} \leq \theta_{G}
$$

Introducing this inequality to our model is straightforward as it simply requires precomputation of shortest paths using Dijkstra algorithm on each demand, which is computationally quite cheap.

\subsection{Infeasible bandwidth/modulation combinations}

As we show here, variables $a_{\ell, b m}$ for some values of $b$ and $m$ can be excluded from the model if the corresponding probabilities $P_{\ell^{b m}}$ violate the required reliability level $F$.

Let us denote by $\bar{F}$ the overall reliability of any feasible solution. We have:

$$
\bar{F}=\prod_{\ell \in L} P_{\ell}^{b_{\ell} m_{\ell}}
$$

where $b_{\ell}$ and $m_{\ell}$ are the selected bandwidth and the modulation, respectively, for link $\ell$. As $\bar{F}$ is a product of probabilities, we can trivially infer that

$$
\bar{F} \leq P_{\ell}^{b_{\ell} m_{\ell}} \quad \forall \ell \in L
$$


Now assume that for some link $\ell$ that $P_{\ell}^{b_{\ell} m_{\ell}}<$ $F$. However for any feasible solution, $\bar{F}$ must be at least equal to $F$, then $P_{\ell}^{b_{\ell} m_{\ell}}<\bar{F}$, which is an obvious contradiction.

Hence, for each link $\ell \in L$ and each, we can exclude without any loss of optimality all bandwidth/modulation pairs $\left(b \in B_{\ell}\right.$ and $\left.m \in M_{\ell}^{b}\right)$ such that $P_{\ell}^{b m}<F$.

\section{Heuristic Lagrangian-based Approach}

We discuss in this section an alternative approach aiming to find high-quality solutions in a short amount of time. This approach features a two-step process:

1. Solve a Lagrangian-relaxed version of the original model to find a tight lower bound (LB), usually unfeasible, on the optimal objective.

2. Design a heuristic-based procedure to move from LB to a nearby feasible solution.

\subsection{First step}

Our Lagrangian-relaxation is based on the dualization of capacity constraints (3). These are obviously the most difficult ones as they associate traffic flows and available capacities on each link. The original objective can be rewritten as:

$$
\begin{aligned}
& \min \sum_{\ell \in L} \sum_{b \in B_{\ell}} \sum_{m \in M_{\ell}^{b}} \operatorname{Cost}_{b} a_{\ell, b m}+ \\
& \sum_{\ell \in L} \mu_{\ell}\left(\sum_{(s, d) \in \mathcal{S D}} \varphi_{\ell}^{s d}-\sum_{b \in B_{\ell}} \sum_{m \in M_{l}^{b}} \theta_{b m} a_{\ell, b m}\right)
\end{aligned}
$$

and then

$$
\begin{aligned}
\min & \sum_{\ell \in L} \sum_{b \in B_{\ell}} \sum_{m \in M_{\ell}^{b}}\left(\text { Cost }_{b}-\mu_{\ell} \theta_{b m}\right) a_{\ell, b m}+ \\
& \sum_{\ell \in L} \sum_{(s, d) \in S D} \mu_{\ell} \varphi_{\ell}^{s d}
\end{aligned}
$$

subject to constraints (2), (4), (5), (6) and (7), and where $\mu_{\ell}$ is a positive Lagrangian multiplier associated to each link.
For a given value of $\mu_{\ell}(\ell \in L)$, this model can be split into two independent parts that can be easily solved. The first part consists in determining for each link $\ell \in L$, the bandwidth/modulation pair $\left(b \in B_{\ell}, m \in M_{\ell}^{b}\right)$ that minimizes Cost $_{b}-\mu_{\ell} \theta_{b m}$ while its corresponding probability $P_{\ell}^{b_{\ell} m_{\ell}}$ is as least equal to $F$. The second part minimizes $\sum_{\ell \in L} \sum_{(s, d) \in S D} \mu_{\ell} \varphi_{\ell}^{s d}$ subject to flow conservation constraints. This is a regular minimum-cost flow problem that can be solved using the classical network simplex algorithm.

Now to solve the whole Lagrangian-relaxed model, good values of multiplier $\mu_{\ell}$ are necessary to provide solution with tight bound to the original problem. Several methods exist in the literature to select and iterate on multiplier. In our case, we use the subgradient algorithm described in many references such as [14].

\subsection{Second step}

As stated below in Algorithm 1, the second step starts from the obtained LB, which probably violates some of the capacity constraints. Our strategy is to increase gradually link capacities until the incumbent solution becomes feasible. First, we sort provisioned links in the LB by decreasing order of their capacities assuming that high-capacity links may convey a large number of requests and hence have more impact. Also, we define two pointers, $h c$ and $l c$, which initially refer to the first and last elements of the sorted list, respectively.

Starting from $h c$, we try at each iteration to increase the capacity of the current link while keeping the cumulative probability (5) below $F$ (RLBC procedure). If the flow constraints are met with this updated link provisioning, then a feasible solution is found (this can be verified by solving a relaxed linear programming version of the original problem). If the capacity of the current link cannot be increased without violating the reliability constraint, then we walk through through the list in the descending order using pointer $l c$ as an attempt to increase the cumulative probability of lower-capacity links without reducing their capacity (RLBF procedure). The rationale behind this is to leave a larger margin for high-capacity links to fit the required reliability by decreasing their modulation. When such link is found, we go back 
to $h c$ to process links in the ascending order. If the two pointers meet and still no feasible solution is found, we reduce iteratively the original demands by a multiplicative factor $\alpha$ until a solution is found.

If the obtained solution entails a significant reduction of transported demands, we repeat the whole process by tweaking randomly the initial sorted link list so that links with equal capacity are processed in a different order.

Finally, we apply a post-processing procedure to try to further reduce the solution cost. This stems from our experimental observations where a significant margin between the cumulative probability of obtained solutions and the required reliability has been noticed. Consequently, the overall cost can be further reduced by substituting some link provisioning by bandwidths that have lower cost and sufficient capacities with respect to carried flows while achieving the required reliability.

\section{Experimental Results}

We present in this section various experimental results of the resolution of our dimensionning problem. First, we compare the original model with the version reinforced with the global capacity valid-inequality and the reduction of the number of variables. Subsequently, we assess the performance of the proposed Lagrangian-based heuristic algorithm in different settings.

Similarly to some previous work [13, 15], our dataset is formed by a number of instances of real-world network topologies and traffic demands proposed by SNDLIB [16]. We consider 3 bandwidths ( $7 \mathrm{MHz}, 14 \mathrm{MHz}, 28 \mathrm{MHz}$ ) having 20, 36 and 48 cost unities, respectively, and 6 modulation schemes (PSK, 16QAM, 32QAM, 64QAM, 128QAM and 256QAM). Probabilities parameters $\left(P_{\ell}^{b m}\right)$ are those defined in [11] using the VigantsBarnett model [17]. Reliability $F$ is initially set to 0.999. The networks instances are described in Table 1. In addition to traffic demands specified in SNDLIB, note that we make a random perturbation up to $5 \%$ on each demand to generate 9 other traffic matrices.

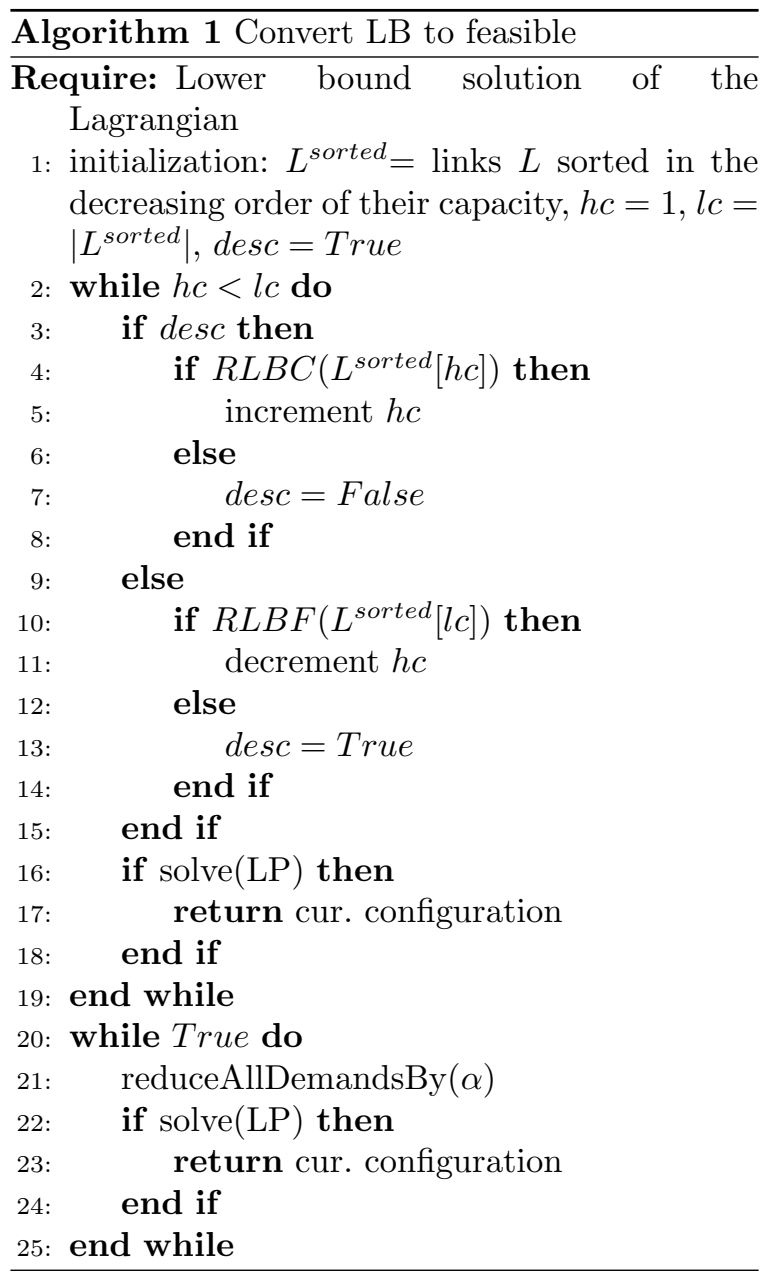

The experiments have been executed on a machine equipped with Intel Xeon E5-2665 processor and 48GB of RAM. We used CPLEX version 12.61 for the resolution of the various MILPs through the Python API. A callback function handled termination if the gap between current incumbent solution and estimated lower bound doesn't decrease during 2 hours.

Table 1 Instances characteristics

\begin{tabular}{llll}
\hline instance & $|V|$ & $|L|$ & $|\mathcal{S D}|$ \\
\hline Di_yuan & 11 & 84 & 22 \\
France & 25 & 90 & 300 \\
Germany50 & 50 & 176 & 662 \\
\hline
\end{tabular}




\subsection{Computational performance of the reinforced models}

We compare here the resolution times of 4 MILP variants: the original model $(\mathrm{OM})$, the original model with valid inequality on global capacity (GCM), the original model with reduction of variables (VRM), the original models with both add-ons (AIM). In the following, we report the resolution time and the gap of the best solution if it is not optimal.

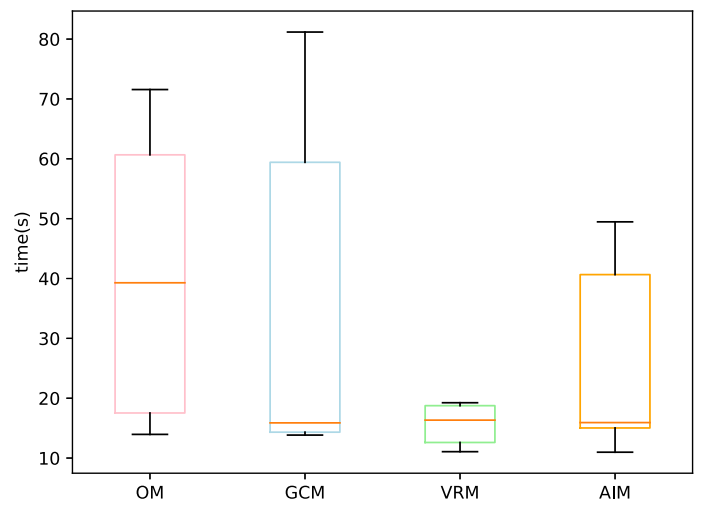

Fig. 1 Resolution times for Di-yuan

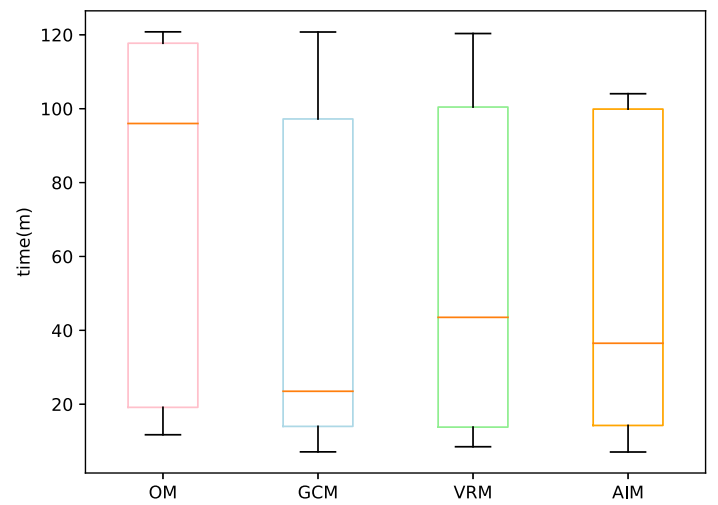

Fig. 2 Resolution times for France

Figures 1, 2 and 3 depict the box plots of resolution time over ten instances of Di-yuan, France and Germany50, respectively. For Di-yuan, where

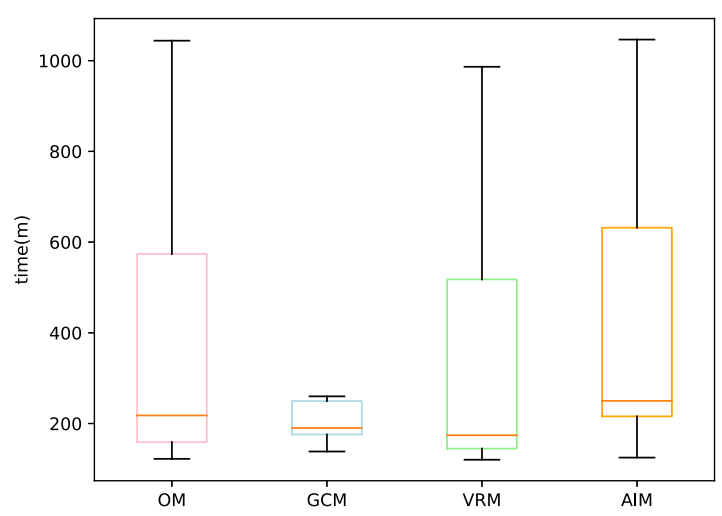

Fig. 3 Resolution times for Germany50

Table 2 BSG for France instances

\begin{tabular}{lllll}
\hline & OM & GCM & VRM & AIM \\
\hline$\# 4$ & $5.2 \%$ & $2.0 \%$ & $3.9 \%$ & $0 \%$ \\
$\# 9$ & $3.9 \%$ & $0 \%$ & $3.9 \%$ & $4.0 \%$ \\
\hline
\end{tabular}

Table 3 BSG for Germany50 instances

\begin{tabular}{lllll}
\hline & OM & GCM & VRM & AIM \\
\hline$\# 0$ & $44.38 \%$ & $20.23 \%$ & $07.25 \%$ & $44.57 \%$ \\
$\# 1$ & $22.99 \%$ & $10.33 \%$ & $21.63 \%$ & $17.57 \%$ \\
$\# 2$ & $20.59 \%$ & $23.01 \%$ & $22.54 \%$ & $43.83 \%$ \\
$\# 3$ & $17.93 \%$ & $20.95 \%$ & $06.69 \%$ & $17.80 \%$ \\
$\# 4$ & $20.32 \%$ & $19.60 \%$ & $22.23 \%$ & $06.98 \%$ \\
$\# 5$ & $18.09 \%$ & $19.21 \%$ & $23.65 \%$ & $06.45 \%$ \\
$\# 6$ & $07.37 \%$ & $18.96 \%$ & $22.24 \%$ & $14.97 \%$ \\
$\# 7$ & $25.99 \%$ & $13.36 \%$ & $09.92 \%$ & $19.51 \%$ \\
$\# 8$ & $20.13 \%$ & $20.89 \%$ & $43.92 \%$ & $22.50 \%$ \\
$\# 9$ & $22.65 \%$ & $43.87 \%$ & $06.67 \%$ & $05.53 \%$ \\
\hline average & $22.04 \%$ & $21.04 \%$ & $18.67 \%$ & $19.97 \%$ \\
\hline
\end{tabular}

all the obtained solutions are optimal, the three reinforced models were able to cut the median time by half . However, VRM showed much less variability that other models. For France, only 8 out of 10 instances have been solved to optimality although the obtained gaps for remaining instances (see Table 2)) are very tight and don't vary significantly from one model to another. In terms of resolution time, GCM exhibits here the best performance as the median has been reduced by about $80 \%$ in comparison to the original model. The situation is different in Germany 50 which is a much larger instance both in terms of links and 
traffic demands. First, no instance has been solved optimally even after several hours of calculations. As reported in Table 3, although VRM returned the tighter average gap, large discrepancies can be noted from one instance to another and from one model to another. For resolution times, RVM resulted in the lowest median by a slim margin, but GCM showed more steadiness over the solved instances. One trend that seemingly stands from these various experiments is that GCM becomes clearly the best option as instances grow in size. For small instances like Di-yuan, RVM appears preferable. In all cases, AIM, which includes all the reinforcement constraints, can be safely dismissed.

\subsection{Performance of the heuristic approach}

We report in this part various results related to the heuristic approach proposed in Section 5 in terms of the resolution time and the solution quality. The reliability level is set to 0.99. Below are the considered performance parameters:

- HRT: resolution time of heuristic method;

- ERT: resolution time of the optimal solution or the best solution given by the MILP in Section 3 ;

- TR: ratio of time reduction between HRT and ERT

- GHS: gap between the heuristic and exact solutions;

- RF: applied reduction factor on traffic.

Table 4 Performance of heuristic solutions of Di-yuan

\begin{tabular}{llllll}
\hline Di-yuan & HRT(s) & ERT(s) & TR & GHS & RF \\
\hline$\# 0$ & 6.57 & 14.72 & $55 \%$ & $9 \%$ & 1.00 \\
$\# 1$ & 5.57 & 19.27 & $71 \%$ & $7 \%$ & 1.00 \\
$\# 2$ & 6.76 & 71.57 & $90 \%$ & $7 \%$ & 1.00 \\
$\# 3$ & 6.07 & 14.28 & $57 \%$ & $7 \%$ & 1.00 \\
$\# 4$ & 6.30 & 16.43 & $61 \%$ & $8 \%$ & 1.00 \\
$\# 5$ & 6.20 & 19.24 & $67 \%$ & $10 \%$ & 1.00 \\
$\# 6$ & 6.58 & 12.54 & $47 \%$ & $8 \%$ & 1.00 \\
$\# 7$ & 7.15 & 10.98 & $34 \%$ & $9 \%$ & 1.00 \\
$\# 8$ & 6.29 & 12.83 & $51 \%$ & $1 \%$ & 1.00 \\
$\# 9$ & 6.18 & 11.08 & $44 \%$ & $7 \%$ & 1.00 \\
\hline Average & 6.36 & 20.29 & $68 \%$ & $7 \%$ & 1.00 \\
\hline
\end{tabular}

Table 5 Performance of heuristic solutions of France

\begin{tabular}{llllll}
\hline France & HRT(s) & ERT(s) & TR & GHS & RF \\
\hline$\# 0$ & 93 & 5175 & $98 \%$ & $14 \%$ & 1.00 \\
$\# 1$ & 91 & 4248 & $97 \%$ & $16 \%$ & 1.00 \\
$\# 2$ & 91 & 424 & $78 \%$ & $19 \%$ & 1.00 \\
$\# 3$ & 101 & 5055 & $98 \%$ & $18 \%$ & 1.00 \\
$\# 4$ & 84 & 6031 & $98 \%$ & $13 \%$ & 1.00 \\
$\# 5$ & 95 & 426 & $77 \%$ & $15 \%$ & 1.00 \\
$\# 6$ & 1849 & 526 & $-71 \%$ & $17 \%$ & 0.95 \\
$\# 7$ & 95 & 886 & $89 \%$ & $17 \%$ & 1.00 \\
$\# 8$ & 98 & 703 & $86 \%$ & $18 \%$ & 1.00 \\
$\# 9$ & 81 & 5608 & $98 \%$ & $15 \%$ & 1.00 \\
\hline Average & 267 & 2908 & $74 \%$ & $16 \%$ & 0.99 \\
\hline
\end{tabular}

Table 6 Performance of heuristic solutions of Germany50

\begin{tabular}{llllll}
\hline Germany & HRT(s) & ERT(s) & TR & GHS & RF \\
\hline$\# 0$ & 3676 & 11639 & $68 \%$ & $24 \%$ & 0.94 \\
$\# 1$ & 1587 & 7618 & $79 \%$ & $23 \%$ & 1.00 \\
$\# 2$ & 2272 & 9092 & $75 \%$ & $23 \%$ & 1.00 \\
$\# 3$ & 2271 & 8300 & $72 \%$ & $22 \%$ & 1.00 \\
$\# 4$ & 3275 & 7212 & $54 \%$ & $21 \%$ & 1.00 \\
$\# 5$ & 4080 & 10354 & $60 \%$ & $25 \%$ & 0.92 \\
$\# 6$ & 1754 & 8806 & $80 \%$ & $24 \%$ & 1.00 \\
$\# 7$ & 2361 & 7327 & $67 \%$ & $23 \%$ & 1.00 \\
$\# 8$ & 3818 & 7494 & $49 \%$ & $22 \%$ & 1.00 \\
$\# 9$ & 1981 & 7872 & $74 \%$ & $24 \%$ & 1.00 \\
\hline Average & 2707 & 8571 & $68 \%$ & $23 \%$ & 0.98 \\
\hline
\end{tabular}

Tables 4, 5 and 6 detail the performance for network instances Di-yuan, France and Germany, respectively. We can see that the heuristic solutions are very close to those found with the exact methods for Di-yuan, less than 20\% for France, and less than 25\% for Germany50. Almost all instances have been solved using the original demand matrix except for one France instance and 2 Germany50 instances, where the original demands have been multiplied by factor ranging from 0.92 to 0.95 .

Figure 4 depicts the impact of required reliability on the total cost for each group of instances. We note similar behavior as the cost starts to increase sharply from 0.994 to 0.995 . However, setting reliability to a very high level induced more additional cost on small instances, e.g., increased by more than $250 \%$ for Di-yuan. Hence, a tradeoff has to be made here by the network operator 
between exploitation cost and availability level offered to users.

Impact of traffic demands on cost is captured in Figure 5. One can notice that reduction in conveyed traffic demand has less impact on total cost for large instances such as Germany50. This could be explained by the larger number of different source-destination demands requiring bandwidth provisioning on a high number of links even if this bandwidth is not fully exploited.

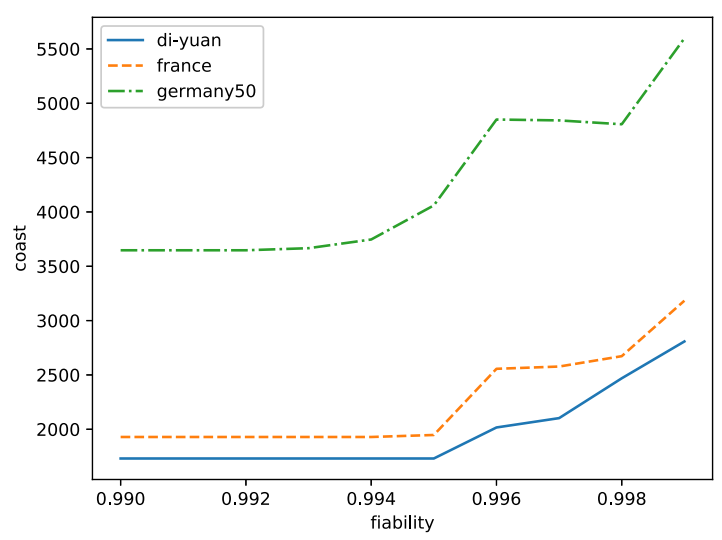

Fig. 4 Impact of reliability on the solution cost

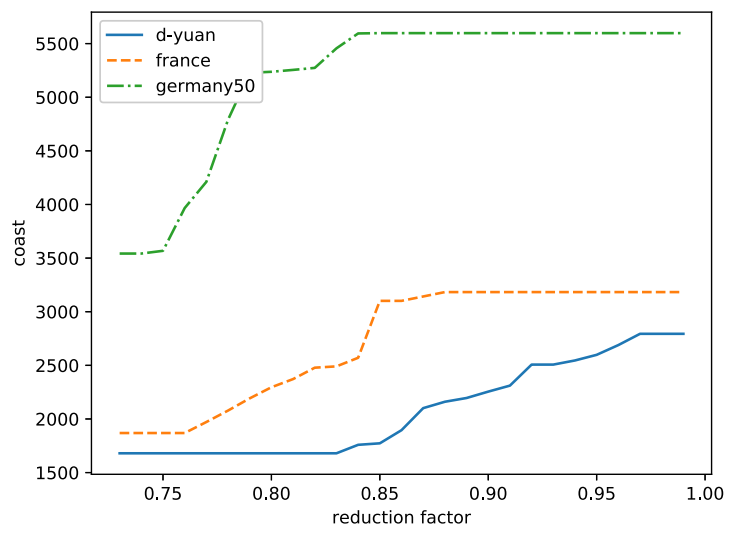

Fig. 5 Impact of demands quantity on the solution cost

\section{Conclusion and Perspectives}

In this paper, we studied a dimensioning problem with bandwidth provisioning and routing optimization in microwave backhaul networks considering statistical distribution of radio conditions. First, we formulated the cost minimization problem as an MILP where the joint probability of used modulation schemes, given the allocated bandwidth on each transmission link, must exceed a prescribed reliability threshold. This model was augmented afterwards to reduce the solution space by characterizing global capacity and eliminating unfeasible combinations of decision variables. Also, we proposed a heuristic approach which starts from the lower bound provided by the Lagrangian relaxation, which is reasonably fast as it separates the original model into two sets of polynomial problems. Then, we designed an algorithm that iterates in an effective way over the provisioned links in order to remove constraint violations by increasing the licensed bandwidth in a conservative manner.

Various numerical results have shown the capacity of our models to solve large instances such as Germany50 in a reasonable amount of time. Furthermore, the heuristic approach succeeded in most experiments to provide solutions within 25\% from exact ones. Our investigations revealed also some interesting relationships between network size on one side, and reliability and traffic requirements on the other. As future work, we plan to add more flexibility in network operations by adjusting dynamically traffic routes according to experienced radio conditions.

\section{References}

[1] Wells, J. (2010) Multi-gigabit Microwave and Millimeter-wave Wireless Communications. Artech House.

[2] Magnanti, T.L., Wong, R.T. (1984). Network design and transportation planning: Models and algorithms. Transportation science 18(1), 1-55 https://doi.org/10.1287/trsc.18. 1.1

[3] Kennington, J.L. (1978). A survey of linear cost multicommodity network flows. Operations Research 26(2), 209-236 https://doi. 
org/10.1287/opre.26.2.209

[4] Minoux, M. (2006). Multicommodity network flow models and algorithms in telecommunications. Handbook of optimization in telecommunications, 163-184 https://doi. org/10.1007/978-0-387-30165-5_7

[5] Bienstock, D., Chopra, S., Günlük, O., Tsai, C.-Y.. (1998). Minimum cost capacity installation for multicommodity network flows. Mathematical programming 81(2), 177-199 https://doi.org/10.1007/BF01581104

[6] Magnanti, T.L., Raghavan, S. (2005). Strong formulations for network design problems with connectivity requirements. Networks: An International Journal 45(2), 61-79 https: //doi.org/10.1002/net.20046

[7] Koster, A.M., Kutschka, M., Raack, C. (2013). Robust network design: Formulations, valid inequalities, and computations. Networks 61(2), 128-149 https://doi.org/10. $1002 /$ net. 21497

[8] Chouman, M., Crainic, T.G., Gendron, B. (2017). Commodity representations and cut-set-based inequalities for multicommodity capacitated fixed-charge network design. Transportation Science 51(2), 650-667 https: //doi.org/10.1287/trsc.2015.0665

[9] Thanh, P.N., Péton, O., Bostel, N. (2010). A linear relaxation-based heuristic approach for logistics network design. Computers \& Industrial Engineering 59(4), 964-975 https: //doi.org/10.1016/j.cie.2010.09.007

[10] Crainic, T.G., Frangioni, A., Gendron, B. (2001). Bundle-based relaxation methods for multicommodity capacitated fixed charge network design. Discrete Applied Mathematics 112(1-3), 73-99 https://doi.org/10.1016/ S0166-218X(00)00310-3

[11] Classen, G., Koster, A.M., Coudert, D., Nepomuceno, N. (2014). Chance-constrained optimization of reliable fixed broadband wireless networks. INFORMS Journal on Computing 26(4), 893-909 https://doi.org/10. 1007/978-3-642-21527-8_5
[12] Coudert, D., Luedtke, J., Moreno, E., Priftis, K. (2018). Computing and maximizing the exact reliability of wireless backhaul networks. Electronic Notes in Discrete Mathematics 64, 85-94 https://doi.org/10.1016/j. endm.2018.01.010

[13] Kodjo, A., Jaumard, B., Nepomuceno, N., Kaddour, M., Coudert, D. (2015). Dimensioning microwave wireless networks. In: Communications (ICC), 2015 IEEE International Conference On, pp. 2803-2809 https://doi. org/10.1109/ICC.2015.7248751. IEEE

[14] Fisher, M.L. (2004). The lagrangian relaxation method for solving integer programming problems. Management science 50(12_supplement), 1861-1871 https://doi. org/10.1002/net.20371

[15] Coudert, D., Nepomuceno, N., Rivano, H. (2010). Power-efficient radio configuration in fixed broadband wireless networks. Computer Communications 33(8), 898-906 https://doi. org/10.1109/ICC.2015.7248751

[16] Orlowski, S., Wessäly, R., Pióro, M., Tomaszewski, A. (2010). Sndlib 1.0-survivable network design library. Networks: An International Journal 55(3), 276-286 https: //doi.org/10.1002/net.20371

[17] Vigants, A. (1975). Space-diversity engineering. Bell system technical journal 54(1), 103-142 https://doi.org/10.1002/j. 1538-7305.1975.tb02828.x

\section{Statements \& Declarations}

- The authors declare that no funds, grants, or other support were received during the preparation of this manuscript.

- The authors have no relevant financial or nonfinancial interests to disclose.

- All authors contributed to the study conception and design. Material preparation, data collection and analysis were performed by Djamil Mehadji. The first draft of the manuscript was written by Djamil Mehadji and Mejdi Kaddour commented on previous versions of the 
manuscript. All authors read and approved the final manuscript. 